\title{
ARROZ E TRIGO: TOLERÂNCIA À SALINIDADE EM SOLUÇÃO NUTRITIVA ( $\left.{ }^{1}\right)$
}

\author{
JOSÉ GUILHERME DE FREITAS $\left.{ }^{2,3}\right)$ \\ e CARLOS EDUARDO DE OLIVEIRA CAMARGO $(2,3)$
}

\begin{abstract}
RESUMO
O estresse hídrico é uma das principais causas da baixa produtividade de grãos nas culturas de arroz e trigo-de-sequeiro no Brasil. Um programa de melhoramento genético usando um método para selecionar plantas tolerantes ao estresse hídrico diminuiria bastante esses efeitos negativos: por isso, tentou-se verificar os comportamentos de cultivares de arroz (sequeiro e irrigado) e trigo sob estresse salino considerando que estes se correlacionassem. Realizaram-se dois experimentos no laboratório da Seção de Arroz e Cereais de Inverno do Instituto Agronômico de Campinas, empregando soluçōes nutritivas. Os cultivares estudados foram os seguintes: arroz-de-sequeiro: IAC-25, IAC-47, IAC-164, IAC-165, IAC-1246, Batatais, Pérola, Pratão Precoce e Dourado Precoce; de arroz irrigado: IAC-120, IAC-435, IAC-899, IR-841, IAC-1278 e IAC-4440; de trigo: IAC-5, IAS-55, Siete Cerros e Paraguay-281. Tanto o arejamento das soluções como a iluminação das plântulas foram contínuos. Usaram-se as concentrações de cloreto de sódio: 0,$0 ; 0,1 ; 0,2 ; 0,3 ; 0,4 ;$ e 0,8 molal $(\mathrm{p} / \mathrm{m})$ ou potenciais osmóticos de 0,$00 ;-0,47 ;-0,93 ;-1,39 ;-1,85$ e $-3,75 \mathrm{MPa}$. As plântulas de arroz cresceram doze dias em solução-tratamento contendo diferentes concentrações de cloreto de sódio em $\mathrm{pH} \mathrm{6,0} \mathrm{e} \mathrm{com} \mathrm{temperatura} \mathrm{de} \mathrm{raizes}$ $30 \pm 1^{\circ} \mathrm{C}$. As plântulas de trigo cresceram em dez dias em soluçăo-tratamento em $\mathrm{pH} 6,0$ e temperatura de raizes $25 \pm 1^{\circ} \mathrm{C}$. Mediu-se o comprimento da major raiz seminal, comprimento da parte aérea (bainha da terceira foIha madura) e pesou-se a matéria seca das partes aéreas e raizes, das plantas dos diferentes cultivarés estudados. A avaliação da diminuiçāo do diâme-
\end{abstract}

(1) Trabalho parcialmente apresentado na IX Reuniāo Latino-Americana de Fisiologia Vegetal, Viçosa, MG, 25-28 de julho de 1983, e na Reuniāo Nacional de Pesquisa de Trigo, Cruz Alta, RS, 16-20 de julho de 1984. Recebido para publicaçao em 24 de abril de 1986 e aceito em 3 de março de 1988.

(2) Seção de Arroz e Cereais de Invemo, instituto Agronômico.

$\left({ }^{3}\right)$ Com bolsa de pesquisa do CNPq. 
tro das raízes e da cor verde foi visual, tanto para o arroz como para o trigo. Os resultados mostraram que o parâmetro da raiz seminal variou mais do que os outros, sendo, portanto, melhor para avaliar a tolerância à salinidade, visando ao melhoramento genético. Assim, com base nas variações das porcentagens diárias médias do comprimento das raízes seminais ao cloreto de sódio, os cultivares de arroz foram classificados em tolerantes: IAC-165, Pratão Precoce, Dourado Precoce, IAC-164 e IAC-120; moderadamente tolerantes: IAC-1246, Pérola, IAC-25, Batatais e IAC-47; moderadamente sensiveis: IAC-899, IAC-435, IAC-4440 e IR-841, e sensivel: IAC-1278. Para o trigo foram considerados como os mais tolerantes os cultivares IAS-55 e Siete Cerros.

Termos de indexaçāo: salinidade, água, potencial osmótico, comprimento radicular e aéreo.

\section{INTRODUÇÃO}

As condições de cultivo de arroz e trigo no Brasil são predominantemente de sequeiro, originando, portanto, instabilidade nos índices de produtividade dessas culturas, decorrentes da grande variação na pluviosidade. As reduçōes no potencial de água do solo devidos à deficiência hídrica podem acarretar periodicamente efeitos negativos no desenvolvimento de plantas cultivadas nessas condiçōes. Para contornar esse problema, a utilização de cultivares de arroz e de trigo tolerantes à seca é uma das estratégias. A adoção de técnicas de laboratório pode ser de grande valia aos programas de melhoramento genético dessas culturas visando à obtenção de cultivares tolerantes à salinidade e, possivelmente, à seca.

Entre os agentes osmolizantes, a solução de cloreto de sódio tem sido usada para induzir o estresse salino em plantas de arroz (PEARSON \& BERNSTEIN, 1959, e TURNER \& MCCAULEY, 1983) e de trigo (SEPASKHAH \& BOERSMA, 1979, e KIRKHAM \& KANEMASU, 1983).

O objetivo deste trabalho foi avaliar o comportamento diferencial de cultivares de arroz e de trigo à salinidade induzida pela adição de cloreto de sódio à solução nutritiva.

\section{MATERIAL E MÉTODOS}

Foram usados os seguintes cultivares:

Arroz: IAC-120, IAC-435, IAC-899, IAC-1278, IAC-4440, IR-841, IAC-25, IAC-47, IAC-164, IAC-165, IAC-1246, Pérola, Dourado Precoce, Pratão Precoce e Batatais.

Trigo: IAC-5, IAS-55, Siete Cerros e Paraguay-281. 
Plantas dos diversos cultivares de arroz e de trigo desenvolveram em solução nutritiva,segundo método descrito por CAMARGO \& OLIVEIRA (1981), e contendo variadas concentraçōes de $\mathrm{NaCl}(0 ; 0,1 ; 0,2 ; 0,3 ; 0,4$ e 0,8 molal, correspondendo, respectivamente, aos potenciais osmóticos de 0,$0 ;-0,47 ;-0,93 ;-1,39$; $-1,86$ e $-3,75 \mathrm{MPa}$.

Os ensaios com arroz e trigo foram conduzidos, não concomitantemente, em câmara de crescimento iluminada continuadamente com luz fluorescente. As soluçōes nutritivas contendo os vários tratamentos de $\mathrm{NaCl}$ (solução tratamento) foram mantidas a $30 \pm 1^{\circ} \mathrm{C}$ e $25 \pm 1^{\circ} \mathrm{C}$, respectivamente para arroz e trigo: antes, ambas cresceram durante 48 horas em água destilada.

Após permanência nas soluções-tratamento por doze dias para o arroz e dez dias para o trigo, avaliaram-se as plântulas (quinze para cada cultivar) quanto ao crescimento da raiz seminal primária (comprimento) e da parte aérea (altura). Em seguida, colocou-se cada plântula (raízes e parte aérea) para secar até peso constante em estufa com temperatura regular para $70^{\circ} \mathrm{C}$, avaliando-as quanto ao acúmulo de massa seca.

Os ensaios foram conduzidos segundo o delineamento de parcelas subdivididas com duas repetiçōes, sendo a parcela principal representada pelas concentrações de $\mathrm{NaCl}$ e as subparcelas, pelos cultivares da espécie em estudo.

\section{RESULTADOS E DISCUSSÃO}

\section{A - ARROZ}

Os cultivares de arroz usados neste trabalho foram de sequeiro e irrigado. Já se sabia, portanto, que os irrigados são mais exigentes em água do que os de sequeiro. Assim, o método usado atendia às necessidades de um bom método de seleção, ou seja: preciso, rápido, passivel de execução e que permitisse selecionar plantas para posteriormente serem transplantadas e conduzidas até completar o seu ciclo.

Pelo quadro 1, verifica-se que o aumento na concentração de cloreto de sódio de zero para 0,1 molal provocou aumento dos comprimento médios das maiores raízes seminais dos cultivares:IAC-1278, IAC-47, IAC-435, Pérola, IAC-25, IAC-164, IAC-120, Pratão Precoce, Dourado Precoce, IAC-165 e Batatais. Todavia, os maiores acréscimos foram verificados nos cultivares de sequeiro IAC-47, Pérola, IAC-25, Pratão Precoce, Dourado Precoce e IAC-165. O IAC-165 apresentou o maior aumento no comprimento da maior raiz seminal para essa concentração de cloreto de sódio. Também houve afinamentos das raízes seminais, ou seja, diminuição dos seus diâmetros para todos os cultivares, mais acentuados para os irrigados. Isso sugere que os cultivares irrigados foram mais sensiveis ao cloreto de sódio e, possivelmente, à diminuiçāo de energia livre da água, confirmando que o crescimento, ou seja, a expansão, é um dos processos fisiológicos 
mais sensiveis ao estresse de água, conforme SEPASKHAH \& BOERSMA (1979) verificaram em trigo. Ainda no quadro 1 , verifica-se que 0 aumento até 0,2 molal na concentração de cloreto de sódio causou um acréscimo nos comprimentos das maiores raizes seminais dos cultivares IAC-164, Pratão Precoce, Dourado Precoce e IAC-165, os quais são recomendados para plantio em condições de sequeiro. Outra vez, o 'IAC-165' exibiu maior aumento no comprimento da maior raiz seminal, apresentando-se o mais verde e de melhor aspecto.

Conforme aumentou a concentração de cloreto de sódio de 0,3 para 0,8 molal, houve uma diminuição no comprimento da maior raiz seminal de todos os cultivares em estudo, quando se comparou com o comprimento em zero molal. Há diferenças na tolerância ao cloreto de sódio entre espécies e pode haver também entre cultivares, conforme LÀZLó et al. (1980) verificaram para Plantago maritima e coronopus. Os resultados obtidos neste trabalho - Quadro 1 - estão de acordo com os de SIEGEL et al. (1980), em milho, SEPASKHAH \& BOERSMA (1979), em trigo, e TURNER \& MCCAULEY (1983), em arroz.

Os cultivares IAC-899, IAC-4440, IAC-435, IR-841 e IAC-1278 foram os que mais diminuiram os comprimentos das maiores raizes seminais, quando a concentração de cloreto de sódio aumentou de zero para 0,2 molal; foram, portanto, os mais sensiveis ao cloreto de sódio e, possivelmente, ao estresse da água em relação aos outros cultivares de arroz estudados. Acima dessa concentração, todos foram considerados sensiveis.

QUADRO 1. Comprimento médio das maiores raízes seminais de quinze cultivares de arroz, medido após 48 horas de crescimento em água destilada, seguido de doze dias de crescimento em soluçōes de tratamento contendo seis diferentes concentrações de cloreto de sódio $(\mathrm{NaCl})$

\begin{tabular}{|c|c|c|c|c|c|c|c|c|c|c|c|c|c|}
\hline \multirow{3}{*}{ Cultivar } & \multicolumn{12}{|c|}{ Concentraçōes de cloreto de sódio (molal) } & \multirow{3}{*}{$\frac{\begin{array}{c}\text { Média } \\
\text { geral }\end{array}}{\%}$} \\
\hline & \multicolumn{2}{|c|}{0,0} & \multicolumn{2}{|c|}{0,1} & \multicolumn{2}{|c|}{0,2} & \multicolumn{2}{|c|}{0,3} & \multicolumn{2}{|c|}{0,4} & \multicolumn{2}{|c|}{0,8} & \\
\hline & $\mathrm{cm}$ & $\%$ & $\mathrm{~cm}$ & $\%$ & $\mathrm{~cm}$ & $\%$ & $\mathrm{~cm}$ & $\%$ & $\mathrm{~cm}$ & $\%$ & $\mathrm{~cm}$ & \% & \\
\hline IAC-899 & 8,12 & 100 & 6,77 & 83 & 4,87 & 60 & 1,60 & 20 & 0,30 & 4 & 0,20 & 2 & 45 \\
\hline IAC-1246 & 14,72 & 100 & 14,16 & 96 & 9,42 & 64 & 0,86 & 6 & 0,50 & 3 & 0,56 & 4 & 46 \\
\hline $1 A C-1278$ & 8,51 & 100 & 10,18 & 120 & 3,04 & 36 & 0,48 & 6 & 0,40 & 5 & 0,46 & 5 & 45 \\
\hline IAC-47 & 11,46 & 100 & 18,25 & 159 & 8,77 & 77 & 0,30 & 3 & 0,40 & 3 & 0,20 & 2 & 58 \\
\hline $1 A C-435$ & 8,97 & 100 & 10,44 & 116 & 4,96 & 55 & 0,16 & 2 & 0,15 & 2 & 0,00 & 0 & 46 \\
\hline $1 A C-4440$ & 9,56 & 100 & 8,73 & 91 & 5,32 & 56 & 1,14 & 12 & 0,35 & 4 & 0,30 & 3 & 44 \\
\hline Pérola & 12,76 & 100 & 15.94 & 125 & 8.56 & 67 & 0,72 & 6 & 0,60 & 5 & 0,56 & 4 & 51 \\
\hline IAC-25 & 11,42 & 100 & 15,28 & 134 & 8,97 & 79 & 0,97 & 8 & 0,60 & 5 & 0,70 & 6 & 55 \\
\hline IR-84i & 8,84 & 100 & 7,21 & 82 & 4,68 & 53 & 1,28 & 14 & 0,30 & 3 & 0,56 & 6 & 43 \\
\hline IAC-164 & 11,35 & 100 & 12,72 & 112 & 11,86 & 104 & 1,42 & 13 & 0,56 & 5 & 0,90 & 8 & 57 \\
\hline $1 A C-120$ & 12,24 & 100 & 14,47 & 118 & 11,47 & 94 & 1,05 & 9 & 0,60 & 5 & 0,56 & 5 & 55 \\
\hline Pratāo Precoce & 9,54 & 100 & 17,55 & 184 & 11,17 & 117 & 0,90 & 9 & 0,96 & 10 & 0,63 & 7 & 71 \\
\hline Dourado Precoce & 8,60 & 100 & 16,95 & 197 & 9,68 & 113 & 1,00 & 12 & 1,30 & 20 & 0,63 & 7 & 75 \\
\hline $1 \mathrm{AC}-165$ & 7,44 & 100 & 13,57 & 182 & 11,05 & 149 & 0,96 & 13 & 0,60 & 8 & 0,50 & 7 & 77 \\
\hline Batatals & 14,28 & 100 & 18,55 & 129 & 11,40 & 80 & 0,86 & 6 & 0,73 & 5 & 0,66 & 5 & 54 \\
\hline Médıas & 13,15 & 100 & 16,48 & 125 & 10,44 & 79 & 1,04 & 8 & 0,56 & 6 & 0,49 & 6 & 55 \\
\hline
\end{tabular}


O comprimento da parte aérea foi medido com base na bainha totalmente desenvolvida na terceira folha do arroz, para coincidir com o inicio do segundo pico de maior sensibilidade ao estresse da água, conforme TURNER \& MCCAULEY (1983). Isso também é válido para o comprimento da maior raiz seminal. À medida que se aumentou a concentração de cloreto de sódio de zero para 0,8 molal, os decréscimos dos comprimentos da parte aérea foram menores para os cultivares IAC-47, Pérola, Dourado Precoce e IAC-165; desse modo, mostraram ser mais tolerantes ao cloreto de sódio na concentração de 0,2. O comprimento da maior raiz seminal, portanto, foi o melhor parâmetro para selecionar plantas mais tolerantes a esse sal, pois no caso de a tolerância ao cloreto de sódio ser devida à permeabilidade da célula da parte aérea, envolveria absorção do cloreto, o que levaria a uma sensivel toxicidade desses ions e desbalanceamento de outros, como os macro e micronutrientes, conforme SIEGEL et al. (1980) verificaram em milho e LÀSLÓ et al. (1980) em plantago.

À medida que se elevou a concentraçāo do cloreto de sódio de zero para 0,1 molal, houve um aumento no peso médio da matéria seca das raizes dos seguintes cultivares de arroz: IAC-899, IAC-4440, Pérola, IR-841, IAC-120, Pratão Precoce e Dourado Precoce. Nos cultivares IAC-164, IAC-25, IAC-165, praticamente não se alterou o peso da matéria seca das raizes, enquanto no 'IAC-1246', 'IAC-1278', 'IAC-47', 'IAC-435' e 'Batatais' diminuiu. Na concentração de 0,2 molal de cloreto de sódio, o 'IAC-25' e o 'IAC-165' tiveram maiores pesos das matérias secas das raízes do que na concentração de zero molal, e 'Dourado Precoce' e 'Pratão Precoce' quase não variaram neste parâmetro; nos outros cultivares, porém, diminuiu o peso da matéria seca das raízes.

O peso médio da matéria seca da parte aérea diminuiu para todos os cultivares, conforme se aumentou a concentração do cloreto de sódio de zero para 0,8 molal, à exceção do 'IAC-165', que manteve o peso em 0,1 molal e o 'IAC-47', que aumentou em 0,2 molal com relação a zero.

Entre os parâmetros estudados, o comprimento da maior raiz seminal parece ser o melhor para selecionar plantas de arroz com tolerância ao cloreto de sódio, pois se baseia no maior comprimento individual. Com relação ao peso médio da matéria seca das raizes e das partes aéreas, esses parâmetros, para serem avaliados, causam a eliminação da planta de arroz, antes de produzir sementes, o que não é desejável em melhoramento genético.

A concentração que permitiu melhor separação entre os cultivares de sequeiro dos irrigados foi de 0,2 molal ou potencial osmótico de -0,93 MPa. Assim sendo, essa técnica parece ser viável para selecionar plantas de arroz mais tolerantes ao cloreto de sódio, quando se baseou no comprimento da maior raiz seminal do arroz (no estádio da terceira para a quarta folha) e quando se utilizaram soluçōes com concentração de 0,2 molal de cloreto de sódio.

$\mathrm{Na}$ escolha dos parâmetros para selecionar os cultivares de arroz mais tolerantes ao cloreto de sódio, utilizou-sə a maior variação de cada parâmetro. $O$ 
comprimento médio das raízes variou de 125 a $6 \%$ e, o das partes aéreas, de 100 a $4 \%$; o peso médio da matéria seca das raizes variou de 100 a $19 \%$ e, o das partes aéreas, de 100 a $15 \%$. Portanto, as variações dos parâmetros foram: 119, 96, 81 e $85 \%$ respectivamente. Por isso, foi escolhido o comprimento médio da raiz seminal como o melhor parâmetro para selecionar plantas de arroz mais tolerantes ao cloreto de sódio, além de permitir que as plantas selecionadas permaneçam vivas e, portanto, produzam sementes, que é uma exigência fundamental no melhoramento genético.

Com base na variação da porcentagem do comprimento das raízes seminais na concentração de 0,2 molal - Quadro 2 - pode-se classificar os cultivares IAC-165, Dourado Precoce, Pratão Precoce, IAC-164 e IAC-120 como tolerantes; IAC-47, IAC-1246, Pérola, IAC-25 e Batatais como moderadamente tolerantes; IAC-899, IAC-435, IAC-4440 e IR-841 como moderadamente sensiveis, e IAC-1278, como o mais sensivel ao cloreto de sódio.

QUADRO 2. Porcentagem do comprimento médio das raizes e classificação com relação à salinidade de quinze cultivares de arroz, medido após 48 horas de crescimento em água destilada, seguido de 12 dias de crescimento em soluções de tratamento contendo seis diferentes concentraçōes de cloreto de sódio $(\mathrm{NaCl})$

\begin{tabular}{|c|c|c|c|c|c|c|c|c|c|c|c|c|c|c|}
\hline \multirow{3}{*}{$\begin{array}{l}\text { Cultivar } \\
\text { IAC-899 }\end{array}$} & \multicolumn{12}{|c|}{ Concentrações de cloreto de sódio (molal) } & \multirow{2}{*}{\multicolumn{2}{|c|}{$\begin{array}{l}\text { Média } \\
\text { geral }\end{array}$}} \\
\hline & \multicolumn{2}{|l|}{0,0} & \multicolumn{2}{|c|}{0,1} & \multicolumn{2}{|l|}{0,2} & \multicolumn{2}{|c|}{0,3} & \multicolumn{2}{|c|}{0,4} & \multicolumn{2}{|c|}{0,8} & & \\
\hline & 100 & $T$ & 83 & $T$ & 601 & MS & 20 & $S$ & 4 & $\mathrm{~S}$ & 2 & $S$ & 45 & MS \\
\hline IAC-1246 & 100 & $\mathrm{~T}$ & 96 & $\mathrm{~T}$ & 641 & MT & 6 & $S$ & 3 & S & 4 & $S$ & 46 & MS \\
\hline IAC-1278 & 100 & $\mathrm{~T}$ & 120 & $T$ & $36 \varsigma$ & $S$ & 6 & $S$ & 5 . & $S$ & 5 & S & 45 & MS \\
\hline IAC-47 & 100 & $\mathrm{~T}$ & 159 & $T$ & 771 & MT & 3 & $S$ & 3 & $\mathrm{~S}$ & 2 & $S$ & 58 & MS \\
\hline IAC-435 & 100 & $\mathrm{~T}$ & 116 & $\mathrm{~T}$ & 551 & MS & 2 & $\mathrm{~S}$ & 2 & $S$ & 0 & $S$ & 46 & MS \\
\hline IAC-4440 & 100 & $\mathrm{~T}$ & 91 & $\mathrm{~T}$ & 561 & MS & 12 & $S$ & 4 & $S$ & 3 & $S$ & 44 & MS \\
\hline Pérola & 100 & $T$ & 125 & $T$ & 671 & MT & 6 & $S$ & 5 & $S$ & 4 & $\mathrm{~S}$ & 51 & MS \\
\hline IAC-25 & 100 & $T$ & 134 & $T$ & 791 & MT & 8 & $S$ & 5 & $S$ & 6 & $S$ & 55 & MS \\
\hline IR-841 & 100 & $\mathrm{~T}$ & 82 & $\mathrm{~T}$ & 53 & MS & 14 & $S$ & 3 & $\mathrm{~S}$ & 6 & $\mathrm{~S}$ & 43 & MS \\
\hline IAC-164 & 100 & $\mathrm{~T}$ & 112 & $\mathrm{~T}$ & 104 & $T$ & 13 & $S$ & 5 & $S$ & 8 & $S$ & 57 & MS \\
\hline $\mid A C-120$ & 100 & $\mathrm{~T}$ & 118 & $\mathrm{~T}$ & 94 & $\mathrm{~T}$ & 9 & $S$ & 5 & $\mathrm{~S}$ & 5 & $\mathrm{~S}$ & 55 & MS \\
\hline Pratão Precoce & 100 & $T$ & 184 & $T$ & 117 & $T$ & 9 & $S$ & 10 & $S$ & 7 & $S$ & 71 & MT \\
\hline Dourado Precoce & 100 & $\mathrm{~T}$ & 197 & $T$ & 113 & $T$ & 12 & $S$ & 20 & $S$ & 7 & $S$ & 75 & MT \\
\hline $\mid A C-165$ & 100 & $T$ & 182 & $T$ & 149 & $T$ & 13 & $S$ & 8 & $S$ & 7 & $S$ & 77 & MT \\
\hline Batatais & 100 & $\mathrm{~T}$ & 129 & $\mathrm{~T}$ & $80 \mathrm{M}^{-}$ & & 6 & $S$ & 5 & $\mathrm{~S}$ & 5 & $\mathrm{~S}$ & 54 & MS \\
\hline Média & 100 & $\mathrm{~T}$ & 125 & $\mathrm{~T}$ & 79 & MT & 8 & $\mathrm{~S}$ & 6 & $S$ & 6 & 3 & 55 & MS \\
\hline
\end{tabular}

$\mathrm{T}=$ tolerante $(>81 \%) ; \mathrm{MT}=$ moderadamente tolerante $(61$ a $80 \%) ; \mathrm{MS}=$ moderadamente sensivel $(41 \mathrm{a}$ $60 \%$ ) e $S=$ sensivel $(<40 \%)$. 


\section{B - TRIGO}

Para o estudo do efeito osmolizante e de sal, há necessidade de que sejam definidos os mecanismos de adaptação vegetal ao efeito da concentração de ions da solução do solo e ao decréscimo da energia livre da água. Para o presente estudo, optou-se entre os mecanismos mais importantes do ponto de vista da produção de grãos de trigo pelo maior comprimento da raiz primária central e parte aérea e a osmorregulação; segundo LEVITT (1972), a diminuição no crescimento é a resposta mais imediata e sensivel a quase todos os estresses. $O$ estresse leva a planta a aumentar a produção de hormônio inibidor do crescimento.

Pelos quadros 3 e 4 - comprimento médio da raiz primária central em relação ao aumento da concentraçāo de cloreto de sódio de 0,0 para 0,1 molal, verifica-se que há variações. Os cultivares IAS-55 e o Siete Cerros apresentaram os maiores valores em relação ao 'IAC-5' e 'Paraguay-281'. Assim, o 'IAS-55' e o 'Siete Cerros' foram considerados tolerantes, e o 'IAC-5' e o 'Paraguay-281', moderadamente tolerantes. Já na concentração de 0,2 molal, o 'IAS- $55^{\prime}$ foi considerado moderadamente tolerante; o 'Siete Cerros', moderadamente sensivel, e o 'IAC-5' e o 'Paraguay-281', sensíveis. Acima da concentração de 0,3 molal, todos os cultivares foram sensiveis ao cloreto de sódio. Uma concentração de cloreto de sódio entre 0,1 e 0,2 molal seria indicada para o estudo diferencial quanto à tolerância a esse sal para os cultivares estudados.

QUADRO 3. Comprimento médio das raízes de quatro cultivares de trigo, medido após 48 horas de crescimento em água destilada, seguido de dez dias de crescimento em soluçöes de tratamento contendo seis diferentes concentraçōes de cloreto de sódio $(\mathrm{NaCl})$

\begin{tabular}{|c|c|c|c|c|c|c|c|c|c|c|c|c|c|}
\hline \multirow{3}{*}{ Cultivar } & \multicolumn{12}{|c|}{ Concentrações de cloreto de sódio (molal) } & \multirow{2}{*}{$\begin{array}{l}\text { Média } \\
\text { geral }\end{array}$} \\
\hline & \multicolumn{2}{|c|}{0,0} & \multicolumn{2}{|c|}{0,1} & \multicolumn{2}{|c|}{0,2} & \multicolumn{2}{|c|}{0,3} & \multicolumn{2}{|c|}{0,4} & \multicolumn{2}{|c|}{0,8} & \\
\hline & $\mathrm{cm}$ & $\%$ & $\mathrm{~cm}$ & $\%$ & $\mathrm{~cm}$ & $\%$ & $\mathrm{~cm}$ & $\%$ & $\mathrm{~cm}$ & $\%$ & $\mathrm{~cm}$ & $\%$ & $\%$ \\
\hline IAC-5 & 16,2 & 100 & 11,1 & 69 & 5,8 & 36 & 3,7 & 23 & 2,8 & 17 & 2,8 & 17 & 44 \\
\hline Siete Cerros & 16,7 & 100 & 16,1 & 96 & 7,5 & 45 & 2,9 & 18 & 1,6 & 10 & 1,5 & 9 & 46 \\
\hline IAS-55 & 13,4 & 100 & 13,6 & 101 & 8,3 & 62 & 3,5 & 26 & 1,8 & 13 & 2,1 & 16 & 53 \\
\hline Paraguay-281 & 15,3 & 100 & 9,8 & 64 & 4,8 & 32 & 3,3 & 21 & 1,6 & 10 & 1,6 & 10 & 40 \\
\hline Média & 15,4 & 100 & 12,7 & 83 & 6,6 & 44 & 3,4 & 22 & 2,0 & 13 & 2,0 & 13 & 46 \\
\hline
\end{tabular}


QUADRO 4. Classificação de quatro cultivares de trigo, com base na porcentagem do comprimento médio das raizes seminais, em soluções nutritivas contendo seis concentrações de cloreto de sódio, mantendo a temperatura das raízes de $25 \pm 1^{\circ} \mathrm{C}$ e pH 6,0

\begin{tabular}{|c|c|c|c|c|c|c|c|c|c|c|c|c|c|c|}
\hline \multirow{2}{*}{ Cultivar } & \multicolumn{12}{|c|}{ Concentraçōes de cloreto de sódio (molal) } & \multirow{2}{*}{\multicolumn{2}{|c|}{$\begin{array}{c}\text { Média } \\
\text { geral }\end{array}$}} \\
\hline & \multicolumn{2}{|l|}{0,0} & \multicolumn{2}{|l|}{0,1} & \multicolumn{2}{|c|}{0,2} & \multicolumn{2}{|l|}{0,3} & \multicolumn{2}{|c|}{0,4} & \multicolumn{2}{|l|}{0,8} & & \\
\hline IAC-5 & 100 & $T$ & 69 & MT & 36 & $\mathrm{~S}$ & 23 & $S$ & 17 & $S$ & 17 & S & 44 & MS \\
\hline Paraguay-281 & 100 & $\mathrm{~T}$ & 64 & MT & 32 & $\mathrm{~S}$ & 21 & $\mathrm{~S}$ & 10 & $\mathrm{~S}$ & 10 & S & 40 & $\mathrm{~S}$ \\
\hline IAS-55 & 100 & $\mathrm{~T}$ & 101 & $\mathrm{~T}$ & 62 & MT & 26 & $S$ & 13 & $\mathrm{~S}$ & 16 & $S$ & 53 & MS \\
\hline Siete Cerros & 100 & $\mathbf{T}$ & 96 & $\mathrm{~T}$ & 45 & MS & 18 & $S$ & 10 & $S$ & 9 & $S$ & 47 & MS \\
\hline Média & 100 & $\mathrm{~T}$ & 82,7 & $T$ & 43,7 & MS & 21,9 & $S$ & 12,6 & $S$ & 13,1 & $S$ & 46 & MS \\
\hline
\end{tabular}

$T=$ tolerante $(>81 \%) ; M T=$ moderadamente tolerante $(61$ a $80 \%) ; M S=$ moderadamente sensivel $(41$ a $60 \%)$ e $S=$ sensivel $(<40 \%)$.

Com o aumento da concentração de $\mathrm{NaCl}$ de zero até 0,2 molal, os comprimentos relativos das raizes primárias centrais (\%) dos cultivares IAS-55, Siete Cerros, IAC-5 e Paraguay-281 diminuiram, respectivamente, de 38, 55, 64 e $68 \%$. Portanto, o aumento na concentração salina e uma diminuição na energia livre da água de 0,93 MPa causaram esses prejuízos aos cultivares estudados; com isso, o IAS-55 foi considerado moderadamente tolerante; o Siete Cerros moderadamente sensivel e o IAC-5 e o Paraguay-281 sensiveis.

O parâmetro comprimento médio da parte aérea não variou praticamente entre os cultivares de trigo na concentração de 0,1 molal de cloreto de sódio. Há menor variação do trigo com relação ao arroz. Isso pode ser explicado por todos os cultivares de trigo serem cultivados tanto em condiçöes de sequeiro como de irrigação. Já para os cultivares de arroz, tal não ocorre; o cultivar ou é de sequeiro ou é irrigado. Além disso, o sistema de irrigaçāo do arroz é por inundaçāo e, o do trigo, por aspersão e, algumas vezes, por banho. A concentração salina nas condições do cultivo do arroz pode variar mais do que nas do trigo. Portanto, há maior variação para o arroz do que para o trigo, com rełação à satinidade; com isso, os critérios de avaliação podem ser diferentes. Na concentração de 0,2 molal, houve maior variação do comprimento médio da parte aérea entre os cultivares de trigo, do que as anteriores, porém insuficiente para uma boa separação deles. Na concentração de 0,3 molal, entretanto, houve maior variação do que nas anteriores. Mas este parâmetro variou menos do que comprimento médio das raízes, o que o torna menos útil nas separaçōes de cultivares mais tolerantes dos menos tolerantes ao cloreto de sódio. 
O parâmetro da matéria seca das raízes varia com o cultivar e concentração de cloreto de sódio, o que indica interação entre cultivar e concentração. De maneira geral, todavia, o cultivar IAS-55 foi o mais tolerante ao cloreto de sódio. O 'Siete Cerros' foi mais tolerante que o 'IAC-5' e 'Paraguay-281'. Outro inconveniente desse parâmetro com relação ao uso no melhoramento genético é a eliminação da planta pára poder avaliá-lo, sem esta produzir sementes. Este variou menos do que o comprimento médio das raízes.

O peso médio da matéria seca da parte aérea aumentou para os cultivares IAC-5, Siete Cerros e IAC-55, e diminuiu para o 'Paraguay-281', na concentração de 0,1 molal de $\mathrm{NaCl}$. Porém, na concentração de 0,2 molal, o mesmo parâmetro aumentou para os cultivares Siete Cerros e IAS-55 e diminuiu para 0 'IAC-5' e 'Paraguay-281', com relação à concentraçāo de zero molal. Além da concentração de 0,3 molal, o peso médio da matéria seca da parte aérea diminuiu para todos os cultivares. Com base na porcentagem média geral, pode-se classificar o 'IAS-55' como tolerante; o 'Siete Cerros', como moderadamente tolerante, e o 'IAC-5' e O 'Paraguay-281', como sensiveis (Quadro 4). Este parâmetro, porém, tem o mesmo inconveniente do anterior: a eliminação da planta antes que produza sementes, ou seja, deixar descendentes para serem testados no melhoramento genético.

\section{CONCLUSŌES}

1) O cloreto de sódio possibilitou a separação dos cultivares de arroz semeados nas condições de sequeiro, dos semeados nas condiçóes de irrigado, em relação à tolerância à salinidade. Os cultivares de arroz-de-sequeiro foram mais tolerantes ao cloreto de sódio.

2) Os cultivares de trigo IAS-55 e Siete Cerros foram mais tolerantes do que o 'IAC-5' e o 'Paraguay-281' ao cloreto de sódio.

3. A melhor concentração de cloreto de sódio para separar os cultivares de arroz de sequeiro dos irrigados foi 0,2 molal. Para o trigo, essa concentração estaria entre 0,1 e 0,2 molal.

4) O comprimento médio das raízes seminais correlacionou melhor a tolerância ao cloreto de sódio para arroz e trigo com o comportamento dessas espécies em condições de sequeiro e irrigado, do que o peso médio da matéria seca das raizes seminais e comprimento e peso médio da matéria seca da parte aérea.

\section{SUMMARY}

\section{TOLERANCE OF RICE AND WHEAT CULTIVARS TO SALINITY}

The water stress and the salinity in the soil are the most important causes of low grain yield of rice and wheat in Brazil, when cultivated on 
upland conditions. Selection for new cultivars showing tolerance to salt effects seems to be one of the methods to solve this problem. The osmotic agent usually used to this purpose is sodium chloride. One experiment was conducted in laboratory conditions using nutrient solutions. The rice genotypes utilized were: upland cultivars IAC-25, IAC-47, IAC-164, IAC-165, IAC-1246, Batatais, Dourado Precoce, Pérola and Pratão Precoce; and irrigated cultivars IAC-120, IAC-435, IAC-899, IAC-1278, IAC-4440 and IR-841. The wheat genotypes utilizated were: upland cultivars IAC-5, IAS-55, Siete Cerros and Paraguay-281. Nutrient solutions aeration and light for the seedlings were provided continuously. Six levels of sodium chloride $(0.0,0.1$, $0.2,0.3,0.4$ and 0.8 molal) or osmotic potentials of $0.00,-0.47,-0.93,-1.39$, -1.85 and $-3.75 \mathrm{MPa}$ were applied int the nutrient solutions. The plastic pots containing the treatment solutions were placed in waterbath with temperature of $30 \pm 1^{\circ} \mathrm{C}$, for rice, and $25 \pm 1^{\circ} \mathrm{C}$, for wheat. The rice and wheat seedlings were cultivated during 48 hours in distilled water and after this period they were changed to pots containing nutrient solutions with different sodium chloride concentrations and $\mathrm{pH}=6.0$. The rice plants were cultivated during twelve days and the wheat plants, ten days. The longest seminal root lengths, the aereal part heights and dry weights of plants were determined. Visual evaluations of root diameter and leaf color were done for all plants in the different treatments. The lenght of the longest seminal root was the best parameter in relation to the others to evaluate the tolerance to salinity for a breeding program. According to the variations in percentage of the longest seminal root lenght, as the sodium chloride concentration increased in the solutions, the rice cultivars IAC-165, Pratão Precoce, Dourado Precoce, IAC-164 and IAC-120 were considered tolerant; IAC-1246, Pérola, IAC-25, Batatais and IAC-47, moderately tolerant; IAC-899, IAC-435, IAC-4440 and IR-841, moderately sensitive and the cultivar IAC-1278, sensitive. The wheat cultivars IAS-55 and Siete Cerros were considered more tolerant than the cultivars IAC-5 and Paraguay-281 up to the concentration of 0.2 molal of sodium chloride. The developed technique was useful to select plants more tolerant to sodium chloride.

Index terms: salinity, water, osmotic potential, root and leaf length.

\section{REFERÊNCIAS BIBLIOGRÁFICAS}

CAMARGO; C.E.O. \& OLIVEIRA, O.F. Tolerância de cultivares de trigo a diferentes niveis de aluminio em soluçôes nutritivas e no soto. Bragantia, Campinas 40.21-31, 1981.

KIRKHAM, M.B. \& KANEMASU, E.T. Wheat. In: TEARE, L.D. \& PEET, M.M. Crop-water relations. New York, John Wiley, 1983. p.481-530.

LÀSLÓ, E.; STUIVER, B \& KUIPER, P.J.C. The effect to salinity on lipid composition and on activity of $\mathrm{Ca}^{2+}$ and $\mathrm{Mg}^{2+}$ stimulated ATPases in salt-sensitive and salt-tolerant. Plantago species. Physiologia Plantarum, Copenhagen, 49.315-319, 1980.

LEVITT, J. Salt and ion stresses. In: RESPONSES of plants environmental stress. New York, Academic Press, 1972. p.489-529. 
PEARSON, G.A. \& BERNSTEIN, L. Salinity effects of several growth stages of rice. Agronomy Journal, Madison, 51:654-657, 1959.

SEPASKHAH, A.R. \& BOERSMA, L. Shoot and growth of $w$ heat seedlings exposed to several levels matric potential and $\mathrm{NaCl}$, induced osmotic potential of soil water. Agronomy Journal, Madison, 71:746-752, 1979.

SIEGEL, S.M.; SIEGEL, B.A.; MASSEY, J.; LAHNE, P. \& CHEN, J. Growth of corn in saline waters. Physiologia Plantarum, Copenhagen, 50:71-73, 1980.

TURNER, F.T. \& MCCAULEY, G.N. Rice. In: TEARE, L.D. \& PEET, M.M. Crop-water relations. New York, John Wiley, 1983. p.481-530. 